ARTICLE

\title{
Optoelectronic mixing with high-frequency graphene transistors
}

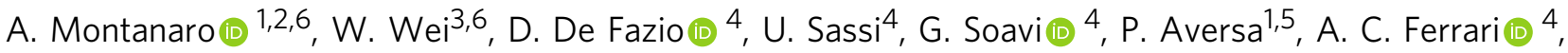 \\ H. Happy $\mathbb{1}^{3}$, P. Legagneux ${ }^{1} \&$ E. Pallecchi (i) ${ }^{3 凶}$
}

Graphene is ideally suited for optoelectronics. It offers absorption at telecom wavelengths, high-frequency operation and CMOS-compatibility. We show how high speed optoelectronic mixing can be achieved with high frequency $(\sim 20 \mathrm{GHz}$ bandwidth) graphene field effect transistors (GFETs). These devices mix an electrical signal injected into the GFET gate and a modulated optical signal onto a single layer graphene (SLG) channel. The photodetection mechanism and the resulting photocurrent sign depend on the SLG Fermi level $\left(E_{F}\right)$. At low $E_{F}$ $(<130 \mathrm{meV})$, a positive photocurrent is generated, while at large $E_{F}(>130 \mathrm{meV})$, a negative photobolometric current appears. This allows our devices to operate up to at least $67 \mathrm{GHz}$. Our results pave the way for GFETs optoelectronic mixers for mm-wave applications, such as telecommunications and radio/light detection and ranging (RADAR/LIDARs.)

\footnotetext{
${ }^{1}$ Thales Research and Technology, Palaiseau, France. ${ }^{2}$ Photonic Networks and Technologies Lab - CNIT, Pisa, Italy. ${ }^{3}$ Univ. Lille, CNRS, Centrale Lille, Univ. Polyechnique Hauts-de-France, UMR8520 - IEMN Institut d'Electronique, de Microélectronique et de Nanotechnologie, Lille, France. ${ }^{4}$ Cambridge Graphene Centre, University of Cambridge, Cambridge, UK. ${ }^{5}$ LSI, CEA/DRF/IRAMIS, Ecole Polytechnique, CNRS, Institut Polytechnique de Paris, Palaiseau, France.

${ }^{6}$ These authors contributed equally: A. Montanaro and W. Wei. ${ }^{凶}$ email: emiliano.pallecchi@univ-lille.fr
} 
$\mathrm{M}$ ixers are a key component of modern communication modules $^{1}$. In telecommunications and in radio detection and ranging (RADAR) systems ${ }^{2}$, the receiver analyzes the modulation of a carrier wave (or waveform) with frequencies in the microwave $(3-30 \mathrm{GHz})$ or $\mathrm{mm}$-wave $(30-300$ $\mathrm{GHz}$ ) range, to extract information 3,4 . As signal processing is performed at near-zero frequencies (baseband ${ }^{3,4}$ ), frequency downconversion is required ${ }^{3,4}$. Downconversion is performed by mixing the modulated high-frequency signal centered around the radio frequency $(\mathrm{RF})$ carrier frequency, $f_{\mathrm{RF}}$, with a local oscillator signal at frequency $f_{\mathrm{LO}}$. This translates the modulation centered around $f_{\mathrm{RF}}$ to $f_{\mathrm{IF}}=f_{\mathrm{LO}}-f_{\mathrm{RF}}$. The local oscillator frequency is typically set near $f_{\mathrm{RF}}$, so that $f_{\mathrm{IF}}$ is close to zero ${ }^{3,4}$. Superheterodyne receivers are a common type of radio receivers using frequency downconversion to process the original signal ${ }^{5}$. For multi-antenna systems, it is preferable to use a single optical signal as a local oscillator and distribute it to each antenna ${ }^{6}$, decreasing the receiver complexity and noise. For this purpose, one option is to use photodetectors (PDs) to transfer the local oscillator signal from the optical to the electrical domain ${ }^{6}$. After that, an electrical mixer is used ${ }^{5}$. A second option is to employ optoelectronic mixers $(\mathrm{OEMs})^{7}$, i.e., PDs capable of mixing optical local oscillator with an electrical signal ${ }^{7}$. OEMs are particularly convenient in RADAR and light detection and ranging (LIDAR) applications ${ }^{7-10}$. State-of-the-art OEMs at $1.55 \mu \mathrm{m}$ are based on III-V semiconductors epitaxially grown on InP ${ }^{11,12}$. These are efficient, but expensive, and can only be heterogeneously integrated in an Si platform ${ }^{11,12}$. Low cost and complementary metal-oxide-semiconductor (CMOS) compatible OEMs require CMOS-compatible materials absorbing light at $1.55 \mu \mathrm{m}^{13}$.

Graphene is promising for optoelectronics ${ }^{14-18}$, with mobilities up to $\sim 150,000 \mathrm{~cm}^{2} \mathrm{~V}^{-1} \mathrm{~s}^{-1}$ at room temperature (RT) ${ }^{19}$, a short ( 1 ps) photocarrier lifetime ${ }^{20-22}$, and a $2.3 \%$ broadband light absorption (including telecom wavelengths) ${ }^{23}$. Graphene-based optoelectronic devices are compatible with Si platforms ${ }^{16,24-27}$. Therefore, graphene-based OEMs could combine telecom operation and CMOS compatibility.

Low frequency $(2 \mathrm{MHz})$ optoelectronic mixing in single-layer graphene (SLG) was studied in ref. ${ }^{28}$ using a transistor structure with an on-chip bias resistor. This reported upconversion of a $2 \mathrm{MHz}$ signal, and downconversion of a $0.45 \mathrm{MHz}$ one, in two types of OEMs consisting of a SLG field-effect transistor (GFET) and a bias resistor. For the first, the oscillating electrical signal was applied to the GFET drain (the optical signal illuminated the GFET channel), whereas in the second the signal was applied to the gate, and the mixing was proportional to the on-chip resistance. A $30 \mathrm{GHz}$ bandwidth (BW) OEM based on SLG was reported in ref. ${ }^{29}$, based on an SLG coplanar waveguide (GCPW) integrating a SLG channel grown by chemical vapor deposition (CVD). The RF signal was injected into the GCPW, whereas a $1.55 \mu \mathrm{m}$ laser illuminated the channel. Optoelectronic mixing was based on the linear dependence of the photocurrent on both optical incident power $\left(P_{\text {opt }}\right)$ and voltage drop $\left(V_{\text {bias }}\right)$ along the channel. As the photocurrent is proportional to $P_{\text {opt }} V_{\text {bias }}{ }^{30}$, upconverted and downconverted signals were generated. This GCPW operated up to $30 \mathrm{GHz}$, with a conversion efficiency, (i.e., ratio of output power at $f_{\mathrm{IF}}$ and input power at $f_{\mathrm{RF}}$ ), of $-85 \mathrm{~dB}$ for a $10 \mathrm{GHz}$ modulated signal. The results in ref. ${ }^{29}$ are far from state-of-the-art OEM performances achieved with III-V semiconductor-based uni-traveling carrier photodiodes: $-22 \mathrm{~dB}$ conversion efficiency at $35 \mathrm{GHz}^{31}$, and $-40 \mathrm{~dB}$ at $100 \mathrm{GHz}^{12}$. However, the CMOS integration of III-V semiconductors is challenging ${ }^{13}$. SLG is CMOS-compatible ${ }^{16}$ but, to technologically bridge the gap with III-V-based OEMs, BW, and conversion efficiency need to be improved ${ }^{29}$. Furthermore, the OEM in ref. ${ }^{29}$ was a two-contact device operating only in the photoconductive regime, at a fixed Fermi level $\left(E_{F}\right)$.

Here, we present a $67 \mathrm{GHz}$ GFET-OEM (three-contact device), exploiting the modulation of $E_{F}$, that controls the photoconductivity. At low (equilibrium) $E_{F}(<130 \mathrm{meV})$ the laser power induces interband transitions ${ }^{32}$, thus the charge carrier density, $n$, and the channel conductance increase (positive photoconductivity). At high $E_{F}(>130 \mathrm{meV})$, the laser heating induces intraband transitions ${ }^{32}$. In this case, the hot carrier distribution reduces the effectiveness of the electronic screening ${ }^{32}$ which, in turn, leads to a higher scattering rate (e.g., owing to Coulomb impurities $^{32}$ or strain disorder ${ }^{33}$ ). The increased scattering decreases the carrier mobility 32 and the resulting photoconductivity is negative ${ }^{32,34-36}$. In our OEMs, an intensitymodulated (up to $67 \mathrm{GHz}$ ) laser beam illuminates part of the SLG channel, generating an AC photocurrent, proportional to the product of optical power and photoresponsivity. An RF signal applied to the gate of $20 \mathrm{GHz}-\mathrm{BW}$ GFETs modulates the photoresponsivity, mixing optical and electrical signals. The performance far exceeds that in ref. ${ }^{29}$. The conversion efficiency $(-67 \mathrm{~dB})$ for a $67 \mathrm{GHz}$ modulated optical signal $\left(f_{\text {opt }}\right)$ is $21 \mathrm{~dB}$ higher than ref. ${ }^{29}$ at $f_{\mathrm{opt}}=10 \mathrm{GHz}$, thanks to the use of an RF GFET with a strong coupling between the input RF signal and SLG (a $0.8 \mathrm{~V}$ signal induces $E_{F} \sim 0.2 \mathrm{eV}$ ). Our results pave the way for the use of graphene in CMOS-compatible OEMs.

\section{Results}

Graphene growth and characterization. SLG is grown via CVD on $35 \mu \mathrm{m}$-thick $\mathrm{Cu}$ foil, following ref. ${ }^{37}$. The temperature, $T$, is raised to $1000^{\circ} \mathrm{C}$ in an $\mathrm{H}_{2}$ atmosphere $(200 \mathrm{mTorr})$, and kept constant for 30 mins. In all, $5 \mathrm{sccm} \mathrm{CH}_{4}$ are then added to the $20 \mathrm{sccm} \mathrm{H}_{2}$ flow to start growth, for additional $30 \mathrm{mins}$ at 300 mTorr. The sample is then cooled at $\sim 1$ mTorr to RT. We use Raman spectroscopy at $514 \mathrm{~nm}$ to characterize the material. Figure la shows the Raman spectrum on $\mathrm{Cu}$ (red line), after $\mathrm{Cu}$ photoluminescence removal ${ }^{38}$. The $\mathrm{D}$ peak is absent, indicating negligible defects ${ }^{39,40}$. The $2 \mathrm{D}$ peak at $22705 \mathrm{~cm}^{-1}$ is a single Lorentzian with full width at half maximum (FWHM) $\sim 31 \mathrm{~cm}^{-1}$, a fingerprint of $S_{L G}{ }^{41}$. The position of the $G$ peak, $\operatorname{Pos}(G)$, is $\sim 1593 \mathrm{~cm}^{-1}$, with FWHM(G) $\sim 12 \mathrm{~cm}^{-1}$. The $2 \mathrm{D}$ to $\mathrm{G}$ intensity and area ratios are $\mathrm{I}(2 \mathrm{D}) / \mathrm{I}(\mathrm{G}) \sim 2.4, \mathrm{~A}(2 \mathrm{D}) / \mathrm{A}(\mathrm{G}) \sim 6.3$.

To prevent ohmic losses at microwave frequencies, a high resistivity $\mathrm{Si}$ wafer $(>8000 \Omega \mathrm{cm})$ covered with $285 \mathrm{~nm} \mathrm{SiO}$ is used. SLG is wet transferred ${ }^{42,43}$ on it as follows. A poly(methyl methacrylate) (PMMA) layer is spin-coated on the surface of SLG/Cu and then placed in a solution of ammonium persulfate (APS) and deionized (DI) water for $\mathrm{Cu}$ etching ${ }^{42}$. The PMMA membrane with attached SLG is then immersed into a beaker filled with DI water for cleaning APS residuals. After, the PMMA/ SLG stack is transferred onto the target substrate and the PMMA layer is removed. SLG is then ion etched to define the channel.

We then characterize via Raman spectroscopy the transferred SLG (blue curve, Fig. 1a). Both measurements on $\mathrm{Cu}$ and $\mathrm{Si}+\mathrm{SiO}_{2}$ are performed under the same conditions of laser power, objective, wavelength, and accumulation time. The Raman signal of SLG on $\mathrm{Cu}$ is more noisy than on $\mathrm{Si}+\mathrm{SiO}_{2}$ due to interference enhancement by the $300 \mathrm{~nm} \mathrm{SiO}$ layer on $\mathrm{Si}^{44,45}$. For SLG on $\mathrm{Si}+\mathrm{SiO}_{2}$ we have $\operatorname{Pos}(\mathrm{G}) \sim 1594 \mathrm{~cm}^{-1}, \operatorname{FWHM}(\mathrm{G}) \sim 11, \operatorname{Pos}(2 \mathrm{D})$ $\sim 2691 \mathrm{~cm}^{-1}, \mathrm{FWHM}(2 \mathrm{D}) \sim 34 \mathrm{~cm}^{-1}, \mathrm{I}(2 \mathrm{D}) / \mathrm{I}(\mathrm{G}) \sim 1.6, \mathrm{~A}(2 \mathrm{D}) /$ $\mathrm{A}(\mathrm{G}) \sim 4.5$. This indicates $\mathrm{p}$-doping $\sim 300 \mathrm{meV}^{46,47}$. I(D)/I(G) $\sim 0.09$ corresponds to a defect density $\sim 4 \times 10^{10} \mathrm{~cm}^{-240,48}$, consistent with what is commonly observed in CVD-SLG ${ }^{49}$. It is possible to improve the process to get a smaller D peak ${ }^{50}$. 

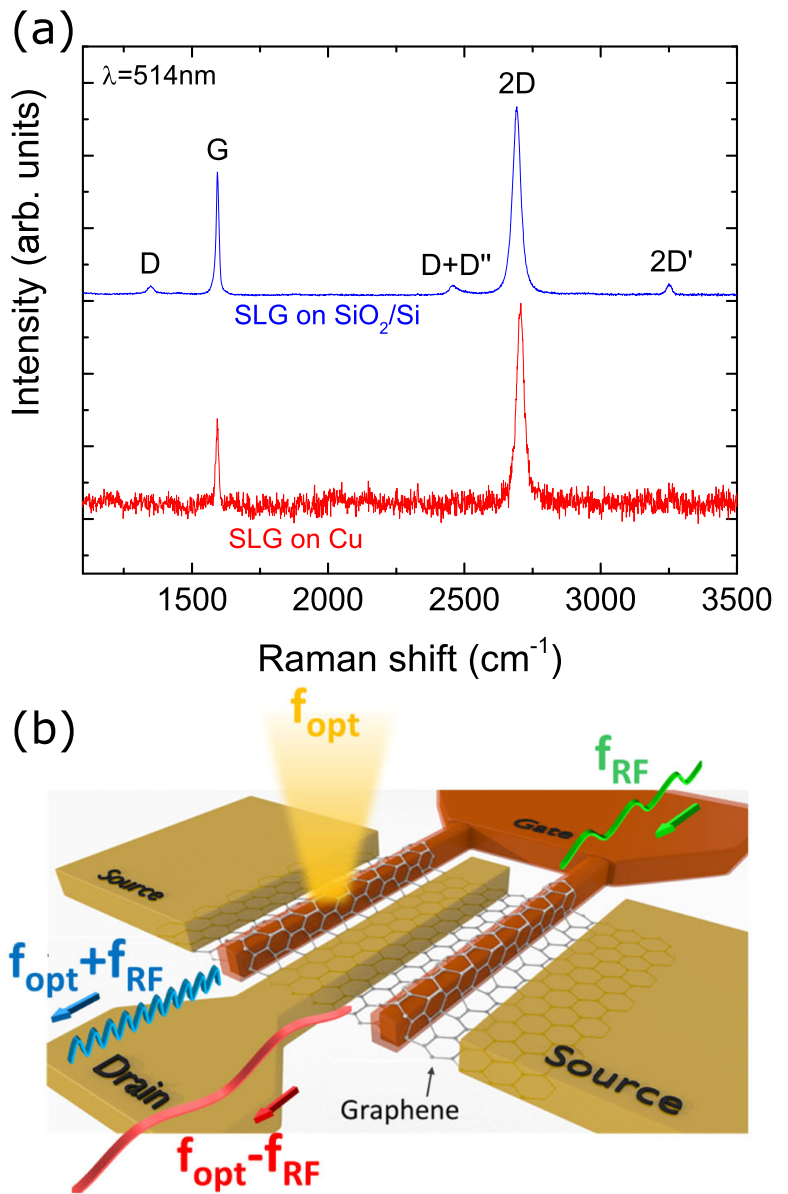

(c)
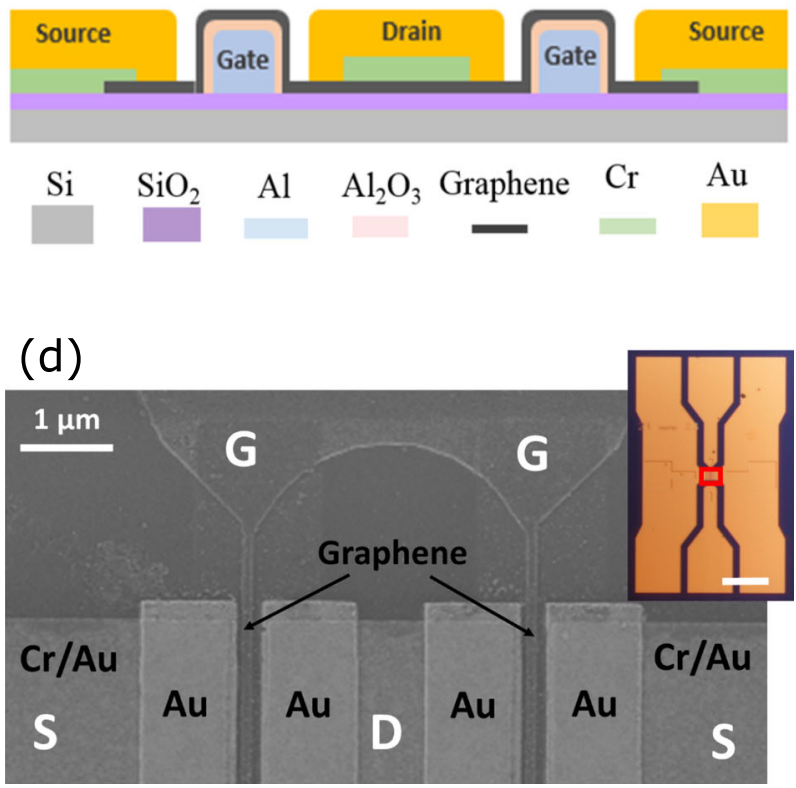

Operational principle and device fabrication. Figure $1 \mathrm{~b}$ is a sketch of our SLG OEM and illustrates its operational principle. It consists of a GFET with a symmetric dual-bottom gate finger. This layout is commonly used for RF applications ${ }^{51}$ and GFETs ${ }^{52}$, and is well suited for GCPWs ${ }^{53}$. Dual-gate finger FETs have a more compact design and a reduced small-signal gate resistance compared with single-gate configurations, for a given equivalent
Fig. 1 SLG Raman characterization and device description. a Representative Raman spectra at $514 \mathrm{~nm}$ of SLG as-grown on Cu (red), and after transfer on $\mathrm{SiO}_{2} / \mathrm{Si}$ (blue). b Principle of operation of our OEM. The mixing of the electrical signal at $f_{\mathrm{RF}}$ with the photodetected signal at $f_{\text {opt }}$ generates two signals at the output (drain): $f_{\mathrm{opt}}+f_{\mathrm{RF}}$ and $f_{\mathrm{opt}}-f_{\mathrm{RF}}$. c Schematic GFET cross-section. The two Al gates (pale blue) are covered by a thin $\mathrm{Al}_{2} \mathrm{O}_{3}$ oxide (pink). SLG (black) is placed on the two gates. The drain and source contacts are $\mathrm{Au}$ (yellow) and $\mathrm{Cr}$ (green). d SEM image of GFET with dualbottom gate finger covered by SLG. The metal in contact with SLG is Au. The inset shows the GFET (red rectangle) integrated into a coplanar waveguide (CPW) (scale bar: $100 \mu \mathrm{m})$.

channel width, resulting in a higher voltage gain ${ }^{54}$. A laser beam is modulated at $f_{\text {opt }}$ and focused on the GFET channel. As a result, a photocurrent that contains an $\mathrm{AC}$ component at $f_{\text {opt }}$ flows through the SLG channel. If a RF signal $f_{\mathrm{RF}}$ is applied to the gate, the output current presents a term at $f_{\mathrm{RF}}$. When both optical and electric signals are applied, the device acts as an OEM: the output contains the product of the two signals, and two AC components at $f_{\text {opt }}+f_{\mathrm{RF}}$ and $f_{\mathrm{opt}}-f_{\mathrm{RF}}$ appear.

A schematic cross-section of the bottom gate GFET is in Fig. 1c. The fabrication starts by patterning the dual-bottom gate finger by e-beam lithography (EBPG 5000 Plus). The gates are made of a $40 \mathrm{~nm}$-thick Al layer deposited by evaporation. A $4 \mathrm{~nm}$ $\mathrm{Al}_{2} \mathrm{O}_{3}$ layer is formed on top of the gates by exposing the substrate to pure oxygen for 30 mins $^{55}$ with an Oxford Plasmalab80Plus at $\sim 100$ mTorr. This thin oxide acts as gate dielectric. The source and drain contacts are made in a two-steps process. First, $\mathrm{Cr} / \mathrm{Au}(5 / 50 \mathrm{~nm})$ pre-contacts are deposited on SLG. Then, ohmic contacts are obtained by placing $30 \mathrm{~nm}$ Au on the $\mathrm{Cr} / \mathrm{Au}-\mathrm{SLG}$ junction. Finally, a $\mathrm{CPW}$ is built with a $\mathrm{Ni} / \mathrm{Au}$ film $(50 / 300 \mathrm{~nm})$. Figure $1 \mathrm{~d}$ is a scanning electron microscopy (SEM) image of the bottom gates covered by SLG. The inset shows a GFET integrated into the CPW. The red square indicates the area occupied by the GFET. The bottom gate GFET design is suitable for OEMs since (1) the SLG channel is on the gate and can be directly illuminated; (2) the use of a thin (4 nm) $\mathrm{Al}_{2} \mathrm{O}_{3}$ dielectric and short gate $(<0.4 \mu \mathrm{m}$ or less) ensures high-frequency operation $^{5,56,57}$.

The device has a cutoff frequency (not de-embedded) $f_{t} \sim$ $25 \mathrm{GHz}$, and a maximum oscillating frequency $f_{\max } \sim 14 \mathrm{GHz}$, as deduced from the S-parameters measured with a Vector Network Analyzer (VNA, Agilent, E8361A). To calibrate the VNA, we use the Line-Reflect-Reflect-Match approach ${ }^{58}$. This allows us to eliminate errors in S-measurements introduced by the environment, such as cables and probe tips used to contact the device under test, and the VNA non-idealities.

Electrical and optoelectronic measurements. The setup in Fig. 2a is used to measure photocurrent and optoelectronic mixing. The output of a $1.55 \mu \mathrm{m}$ distributed feedback laser is modulated by a Mach Zehnder modulator in the double sideband suppression carrier mode ${ }^{59}$, to obtain a modulated beam at $f_{\text {opt }}$. This is then amplified with an Erbium-doped fiber amplifier. The maximum $f_{\text {opt }}$ that our setup can probe is $67 \mathrm{GHz}$. The diameter of the focused laser spot is $\sim 2 \mu \mathrm{m}$ (inset of Fig. 2a). The maximum power impinging on the sample is $\sim 60 \mathrm{~mW}$, which corresponds to $\sim 20 \mathrm{~mW} / \mu \mathrm{m}^{2}$. The gate and drain are connected to a VNA with two high-frequency $(67 \mathrm{GHz})$ air coplanar probes. Bias tees are used to add a DC bias to channel and gate electrodes, and to measure the DC currents and voltages with a Source-MeasureUnit (Keithley 2636B). After illuminating the device, we verify the stability of the signal before measuring the RF photocurrent, whereas monitoring the DC value of the channel resistance, to 

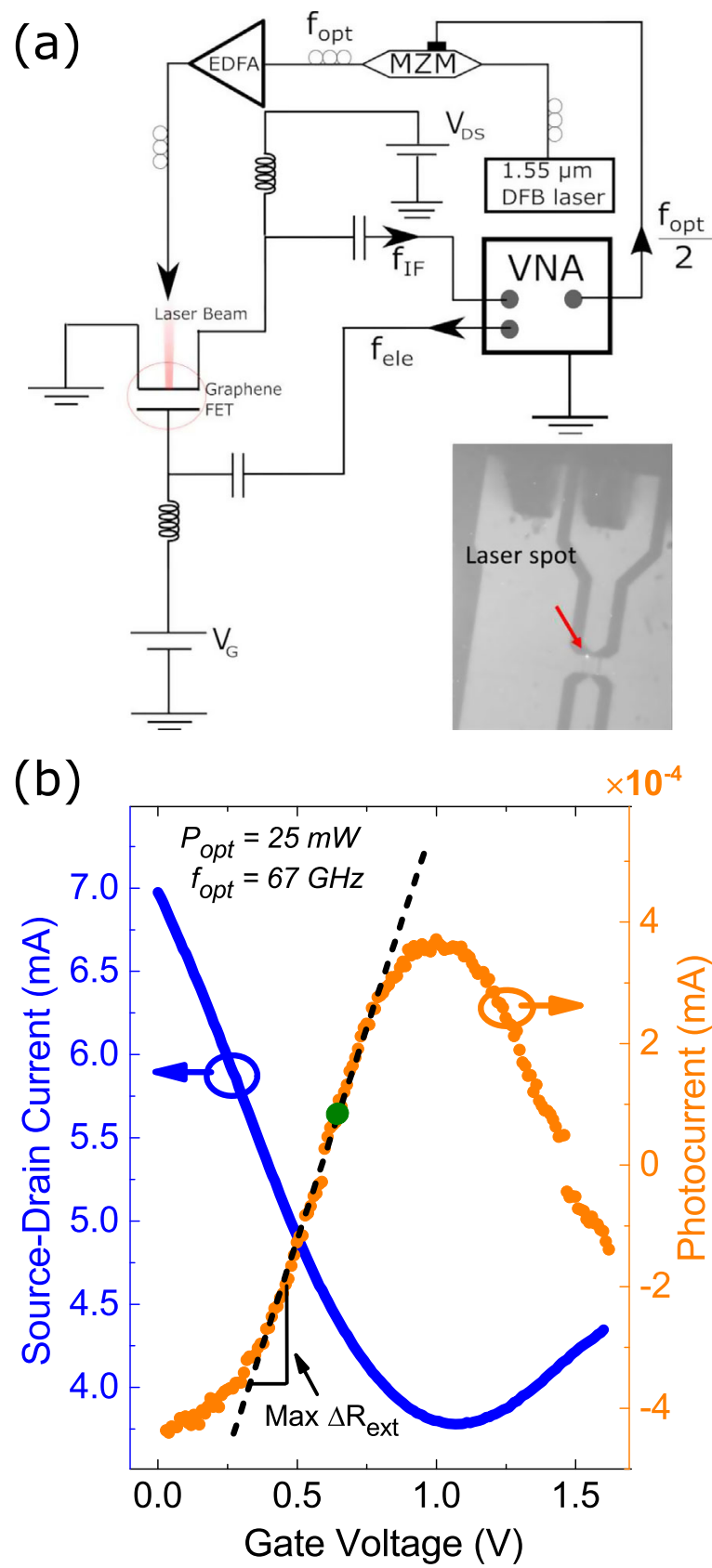

Fig. 2 Measurement setup and device characteristics. a Experimental setup: a CW laser is modulated via a MZM. It is then amplified with an EDFA and focused on the GFET. An AC signal is applied to the gate. The output $f_{I F}$ is measured on a VNA. Inset: optical image of device with laser focused on the channel. b Blue curve: source-drain current versus gate voltage, for $V_{D S}=200 \mathrm{mV}$. Orange curve: photocurrent versus gate voltage, generated by a $25 \mathrm{~mW}$ beam focused on the SLG channel.

ensure that no damage nor significant modification is induced by the laser power or DC bias. We do not observe any degradation or time-dependent drift in the DC or RF currents over a period of at least $3 \mathrm{~h}$, the typical measurement time.

We now present the results for a representative OEM with SLG channel width $W=24 \mu \mathrm{m}$, length $L=400 \mathrm{~nm}$, and gate length $L_{G}=200 \mathrm{~nm}$. The blue curve in Fig. $2 \mathrm{~b}$ is the source-drain current, $I_{\mathrm{DS}}$, as a function of gate voltage, $V_{\mathrm{GS}}$, at $V_{\mathrm{DS}}=200 \mathrm{mV}$, which shows the typical ambipolar conduction behavior of a GFET (i.e, electrical conductivity due to electrons/holes $(e / h)$, depending on the position of $E_{F}$ with respect to the charge neutrality point, $\mathrm{CNP})^{60}$. The minimum conductance is reached at $V_{\mathrm{GS}}=1.1 \mathrm{~V}$, which corresponds to the CNP voltage $\left(V_{\mathrm{CNP}}\right)$. When $V_{\mathrm{GS}}$ increases (decreases) with respect to $V_{\mathrm{CNP}}$, the $e$ (h) density increases, leading to a reduction of channel resistivity, so an increase of the current flowing in the channel ${ }^{60} . \mu$ is calculated as $\mu=L g_{m} /\left(\mathrm{W} \cdot \mathrm{C}_{G} \mathrm{~V}_{\mathrm{DS}}\right)^{61}$. The transconductance $\mathrm{g}_{m}=d I_{\mathrm{DS}} / d V_{\mathrm{GS}}{ }^{13}$ is obtained from the transfer characteristic $I_{\mathrm{DS}}\left(V_{\mathrm{GS}}\right)$ at $\mathrm{V}_{\mathrm{DS}}=10 \mathrm{mV}$. The gate capacitance is $C_{o x}$ is $\sim 5 \mathrm{fF} / \mu \mathrm{m}$, obtained from $S$ parameters measurements on 60 devices of the same kind ${ }^{55}$. We get $\mu \sim 2500 \mathrm{~cm}^{2} \mathrm{~V}^{-1} \mathrm{~s}^{-1}$, consistent with that of non-encapsulated CVD-SLG ${ }^{37}$.

We now consider the photoresponse. The OEM is biased at $V_{\mathrm{DS}}=200 \mathrm{mV}$ and illuminated with a laser modulated at $f_{\mathrm{opt}}=$ $67 \mathrm{GHz}$. The electrical power $P_{\mathrm{RF}}$ measured by the VNA is used to derive the photocurrent $I_{\mathrm{ph}}$. From Joule's law ${ }^{13} I_{\mathrm{ph}}=\sqrt{\frac{P_{\mathrm{RF}}}{Z_{\mathrm{VNA}}}}$, with $Z_{\mathrm{VNA}}=50 \Omega$ the VNA input impedance. The use of bias tees allows us to simultaneously measure the DC component, i.e., the dark current (blue curve in Fig. 2b) and the AC component, i.e., the photocurrent (orange curve in Fig. 2b) as a function of $V_{\mathrm{GS}}$, for a $25 \mathrm{~mW}$ incident optical power. The photocurrent, $I_{\mathrm{ph}}$, sign depends on $\mathrm{V}_{\mathrm{Gs}} . I_{\mathrm{ph}}$ is positive and has a local maximum close to the CNP. At low (equilibrium) $E_{F}(<130 \mathrm{meV})$, the laser power induces interband heating ${ }^{32}$, thus an increase of $n$ (positive photoconductivity). Therefore, the photocurrent has the same sign as the DC current in the channel, owing to the DC bias. At high $E_{F}>130 \mathrm{meV}$, the sign of the photocurrent is opposite to the DC current (negative photoconductivity). In this case, laser heating induces intraband transitions, which lead to a reduction of electronic screening of the long-range Coulomb interaction between SLG's carriers and charged impurities in the substrate 22,32 . The $E_{F}$ at which the transition between positive and negative photocurrent takes place is $\sim 0.1-0.2 \mathrm{eV}^{32,34}$. The value depends on the charge transport scattering rate in SLG, i.e., the mean time interval between two collisions in the diffusive transport picture ${ }^{62}$, and on the charge neutrality region width ${ }^{63}$ (see Methods). In our experiment, we observe this transition at $\sim 130 \mathrm{meV}$.

The external photoresponsivity, $R_{\text {ext }}$, is defined as ${ }^{15,64} R_{\text {ext }}=$ $\frac{\left|I_{\mathrm{ph}}\right|}{P_{\mathrm{c}} \mathrm{w}_{\text {eff }}}$, with $P_{\mathrm{c}} \mathrm{w}_{\text {eff }}=31 \% P_{\mathrm{cw}}$ the fraction of the optical power coupled to the SLG channel. We get $R_{\text {ext }} \sim 0.22 \mathrm{~mA} / \mathrm{W}$. For $V_{\mathrm{GS}}=0 \mathrm{~V}$, the device reaches its maximum $I_{\mathrm{ph}} \sim-4.2 \times 10^{-4} \mathrm{~mA}$ and the photocurrent generated by a $67 \mathrm{GHz}$ laser modulation is measured as a function of DC bias and optical power.

Figures $3 \mathrm{a}, \mathrm{b}$ plot the photocurrent as a function of DC bias at $P_{\mathrm{opt}}=40 \mathrm{~mW}$ and as a function of the optical power for $V_{\mathrm{DS}}=$ $330 \mathrm{mV}$. The response is linear in both cases, as expected for a photoconductor ${ }^{34}$. The frequency response of the photodetected power is then measured as a function of $f_{\text {opt}}$, Fig. $4 \mathrm{a}$. We get a flat response over the whole band that can be investigated by our VNA, showing that the intrinsic photodetection BW is $>67 \mathrm{GHz}$. The photocurrent can originate from the SLG channel located above the gate, or from the SLG-metal contacts that are not gated (see Fig. 1c). As the photoresponse strongly depends on $\mathrm{V}_{\mathrm{GS}}$, as shown in Fig. 2b, we ascribe it to the illuminated part of the SLG channel located above the gate. If both contacts are illuminated, the photocurrents are opposite and cancel out. If the photocurrent originates mainly from one SLG-metal contact, the photocurrent at $V_{\mathrm{DS}}=0 \mathrm{~V}$ should be significant. $R_{\text {ext }} \sim 0.8 \mathrm{~mA} / \mathrm{W}$ was reported in ref. 65 for a detector exploiting the metal-SLG contacts, at $V_{\mathrm{DS}}=0 \mathrm{~V}$. As seen in Fig. 3, at $V_{D S}=0 \mathrm{~V}$ the photocurrent is negligible. We also rule out possible asymmetric heating effects on the channel, owing to beam location, by performing a photocurrent measurement as a function of laser 

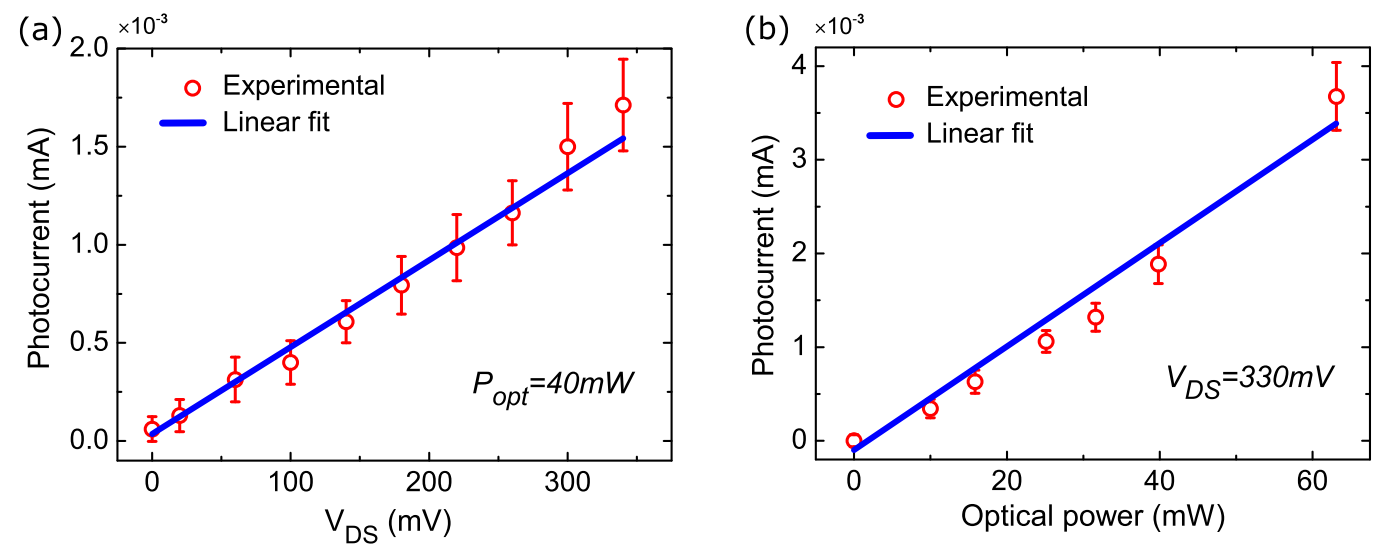

Fig. 3 Photocurrent measurements. a Photocurrent as a function of $V_{D S}$ at $40 \mathrm{~mW}$ optical power, $\mathbf{b}$ photocurrent as a function of the optical power, at $V_{D S}=330 \mathrm{mV}$. Error bars are obtained from the VNA measurement noise standard deviation.
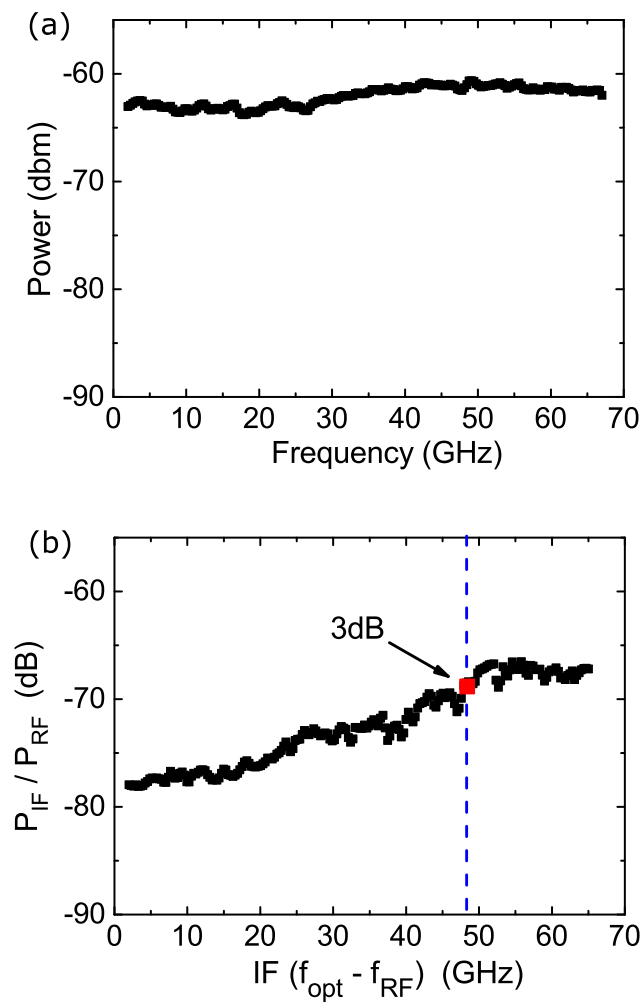

Fig. 4 RF Optoelectronic characterization. a Maximum photodetected power at $V_{\mathrm{GS}}=0 \mathrm{~V}, V_{\mathrm{DS}}=330 \mathrm{mV}$, as a function of $f_{\mathrm{opt}}$. $\mathbf{b} P_{\mathrm{IF}} / P_{\mathrm{RF}}$ at $V_{\mathrm{GS}}=$ $0.6 \mathrm{~V}, V_{D S}=330 \mathrm{mV}$. Optical power in $\mathbf{a}, \mathbf{b}$ is $60 \mathrm{~mW}$.

spot position. We observe a very weak response at $V_{\mathrm{DS}}=0 \mathrm{~V}$, regardless of laser spot position, as shown in Methods, Fig. 10. Thus, the role of contacts can be neglected.

In order to operate the device as an OEM (instead of a PD), an RF signal $f_{\mathrm{RF}}$ is added to the DC gate, Fig. $1 \mathrm{~b}$. $f_{\text {opt }}$ is maintained at $67 \mathrm{GHz}$, whereas $f_{\mathrm{RF}}$ is swept between 2 and $65 \mathrm{GHz}$. A VNA is used to record $P_{\mathrm{IF}}$ and the transistor power at the intermediate frequency $f_{\mathrm{IF}}=f_{\mathrm{opt}}-f_{\mathrm{RF}}$.

An important parameter for OEMs is the downconversion efficiency ${ }^{5} P_{\mathrm{IF}} / P_{\mathrm{RF}}$, with $P_{\mathrm{RF}}$ the power at the source and $P_{\mathrm{IF}}$ that measured at the VNA. For our device, the maximum $P_{\mathrm{IF}} / P_{\mathrm{RF}}$ is $-67 \mathrm{~dB}$ at $V_{\mathrm{GS}}=0.6 \mathrm{~V}$. For this $V_{\mathrm{GS}}$, Fig. $4 \mathrm{~b}$ plots $P_{\mathrm{IF}} / P_{\mathrm{RF}}$ as a function of $f_{\mathrm{IF}}$. The $\mathrm{BW}$ and $P_{\mathrm{IF}} / P_{\mathrm{RF}}$ of our OEMs far exceed $\left(+37 \mathrm{GHz}\right.$ in $\mathrm{BW}$ and 2 orders of magnitude in $\left.P_{\mathrm{IF}} / P_{\mathrm{RF}}\right)$ those of ref. ${ }^{29}$, where the input RF signal modulates the SLG bias (resistive coupling), thus the photocurrent amplitude. In our OEMs, the input RF signal is coupled to SLG via the gate oxide (capacitive coupling), which results in a modulation of $E_{F}$ and, consequently, in a change of the photocurrent mechanism and sign. These high $\mathrm{BW}$ and $P_{\mathrm{IF}} / P_{\mathrm{RF}}$ come from the strong coupling (i.e., strong electric field for a small applied voltage, $0.25 \mathrm{~V} / \mathrm{nm}$ ) between the input RF signal (applied to the Al back-gate) and the SLG channel, thanks to the use of a $\sim 4 \mathrm{~nm}$ oxide. As a consequence, an efficient field effect is achieved ${ }^{60}$. A signal with an amplitude $\sim 0.8 \mathrm{~V}$ induces a $E_{F}$ modulation $\sim 0.2 \mathrm{eV}$. Thus, a small signal $(0.8 \mathrm{~V})$ is needed to obtain optoelectronic mixing. The high-frequency operation of the GFET $(\sim 20 \mathrm{GHz}, 3 \mathrm{~dB} \mathrm{BW}$, Fig. $4 \mathrm{~b})$ comes from the short channel length $(400 \mathrm{~nm})$ and the small gate capacitance $C_{\mathrm{ox}} \sim 60 \mathrm{fF}^{66}$.

Figure $5 \mathrm{a}$ is a color map of the $67 \mathrm{GHz}$ photocurrent as a function of $V_{\mathrm{GS}}, V_{\mathrm{Ds}}$. We then add to the DC gate bias an electrical signal at $10 \mathrm{GHz}$. The resulting downconverted photocurrent at $f_{\mathrm{IF}}=57 \mathrm{GHz}$ is plotted as a function of $V_{\mathrm{DS}}, V_{\mathrm{GS}}$ in Fig. $5 \mathrm{~b}$. By differentiating the map in Fig. 5a with respect to $V_{\mathrm{GS}}$, we obtain Fig. 5c, which resembles Fig. 5b. This is best seen in Fig. 6, which plots both values as a function of $V_{\mathrm{GS}}$ for $V_{\mathrm{DS}}=200 \mathrm{mV}$. The curves of the downconverted photocurrent and of the derivative of the photocurrent can be superposed. This result is valid regardless of frequency, see Methods Fig. 9.

\section{Discussion}

This behavior can be explained by a small-signal analysis.

Let us consider the modulated optical power impinging on the $\mathrm{PD}, P_{\mathrm{opt}}=P_{\mathrm{cw}}+P_{\mathrm{mod}} \sin \left(2 \pi f_{\mathrm{opt}} t\right)$, with $P_{\mathrm{mod}}$ the amplitude of the varying part of the optical power. The photocurrent is proportional to $P_{\mathrm{opt}}$ through the factor $R_{\text {ext }}$. This depends on $V_{\mathrm{GS}}$, as for Fig. $2 \mathrm{~b}$, and is almost independent on $f_{\text {opt }}$, Fig. $4 \mathrm{a}$. Therefore, the photocurrent can be written as:

$$
I_{\mathrm{ph}}\left(V_{\mathrm{GS}}\right)=R_{\mathrm{ext}}\left(V_{\mathrm{GS}}\right)\left[P_{\mathrm{cw}}+P_{\mathrm{mod}} \sin \left(2 \pi f_{\mathrm{opt}} t\right)\right]
$$

By applying to the gate a DC bias $\bar{V}_{\mathrm{GS}}$ and a small signal $\delta_{V_{\mathrm{GS}}} \sin \left(2 \pi f_{\mathrm{RF}} t\right)$, we get:

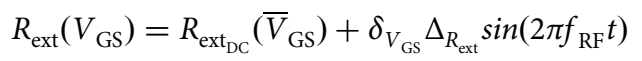

where

$$
\Delta_{R_{\text {ext }}}=\left.\beta\left(f_{\mathrm{RF}}\right) \frac{d R_{\mathrm{ext}}\left(V_{\mathrm{GS}}\right)}{d V_{\mathrm{GS}}}\right|_{V_{\mathrm{GS}}=\bar{V}_{\mathrm{GS}}}
$$

We include dependence on injected electrical frequency through a frequency-dependent proportionality constant $\beta\left(f_{\mathrm{RF}}\right)$. The total 

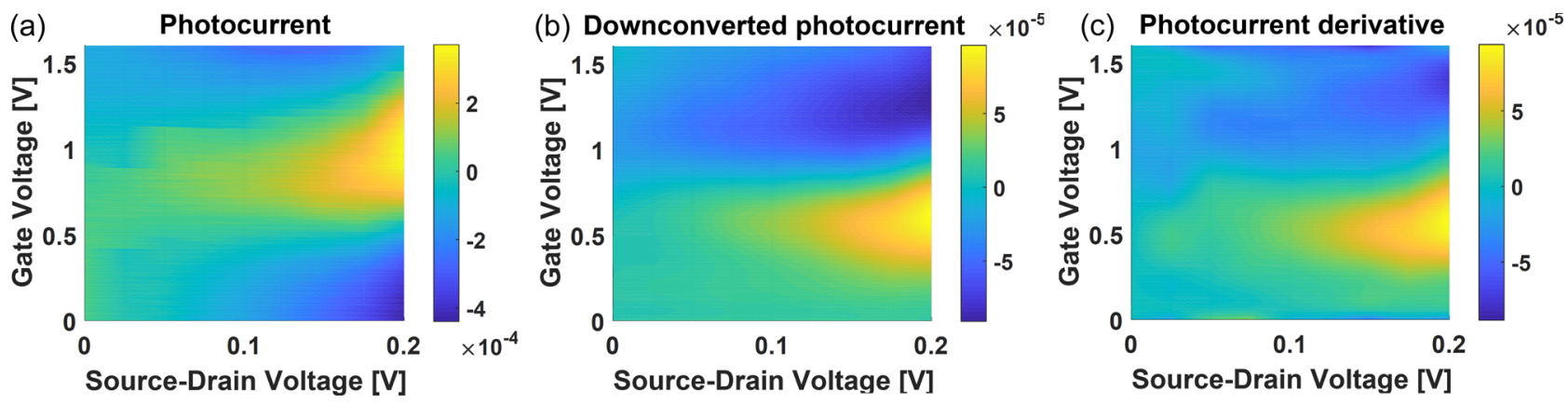

Fig. $5 \mathbf{V}_{\mathbf{G S}}-\boldsymbol{V}_{\mathbf{D S}}$ maps. a Photocurrent map as a function of $V_{\mathrm{GS}}, V_{\mathrm{DS}}$. b Downconverted photocurrent map as a function of $V_{\mathrm{GS}}, V_{\mathrm{DS}}$. $\mathbf{c}$ Derivative of a with respect to $V_{G S}$. The photocurrent values are in $\mathrm{mA}$.

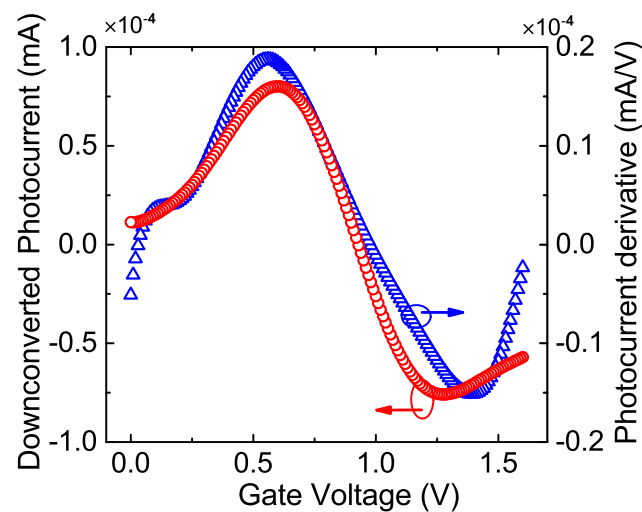

Fig. 6 Downconversion efficiency vs $\boldsymbol{V}_{\mathbf{G S}}$. Red curve: cut of Fig. $5 \mathrm{c}$ for $V_{D S}=200 \mathrm{mV}$. Blue curve: cut of Fig. $5 b$ for $V_{D S}=200 \mathrm{mV}$.

photocurrent has four terms:

$$
\begin{aligned}
I_{\mathrm{ph}}= & R_{\mathrm{ext}_{\mathrm{DC}}}\left(\bar{V}_{\mathrm{GS}}\right) P_{\mathrm{cw}}+\delta_{V_{\mathrm{GS}}} \Delta_{R_{\mathrm{ext}}} P_{\mathrm{cw}} \sin \left(2 \pi f_{\mathrm{RF}} t\right) \\
& +R_{\mathrm{ext}_{\mathrm{DC}}}\left(\bar{V}_{\mathrm{GS}}\right) P_{\mathrm{mod}} \sin \left(2 \pi f_{\mathrm{opt}} t\right) \\
& +\delta_{V_{\mathrm{GS}}} \Delta_{R_{\mathrm{ext}}} P_{\mathrm{mod}} \sin \left(2 \pi f_{\mathrm{RF}} t\right) \sin \left(2 \pi f_{\mathrm{opt}} t\right)
\end{aligned}
$$

The first is the DC photocurrent. The second describes the DC photocurrent modulated by the electrical signal. The third represents the photocurrent modulated at $f_{\text {opt }}$, Fig. 2b. The fourth describes the optoelectronic mixing and can be rewritten as:

$$
\begin{aligned}
\delta_{V_{\mathrm{GS}}} \Delta_{R_{\mathrm{ext}}} P_{\mathrm{mod}} \sin \left(2 \pi f_{\mathrm{RF}} t\right) \sin \left(2 \pi f_{\mathrm{opt}} t\right)= & \frac{1}{2} \delta_{V_{\mathrm{GS}}} \Delta_{R_{\mathrm{ext}}} P_{\mathrm{mod}}\left\{\cos \left[2 \pi\left(f_{\mathrm{RF}}-f_{\mathrm{opt}}\right) t\right]\right. \\
& \left.+-\cos \left[2 \pi\left(f_{\mathrm{RF}}+f_{\mathrm{opt}}\right) t\right)\right\}
\end{aligned}
$$

Equation (5) has two components at $f_{\mathrm{opt}}+f_{\mathrm{RF}}$ and $f_{\mathrm{IF}}=f_{\mathrm{opt}}-f_{\mathrm{RF}}$. It shows that the mixed signal depends exclusively on $\Delta_{R_{\text {ext }}}$ i.e., on the derivative of $R_{\text {ext }}$ with respect to $V_{\mathrm{GS}}$, not on $R_{\text {ext }}$ itself, in accordance with Fig. 5. $\Delta_{R_{\text {ext }}}$ is maximum for $V_{\mathrm{GS}} \sim 0.6 \mathrm{~V}$, Fig. 6, i.e., in a region where the photocurrent changes sign, indicated in Fig. $2 \mathrm{~b}$ with a green dot. The sharper is the transition between the two competing phenomena (photoconductive and bolometric) generating the photocurrent, the higher is $\Delta_{R_{\text {ext }}}$ and the optoelectronicmixing efficiency.

Since $\mathrm{R}_{\mathrm{ext}}$ is proportional to the photoconductivity $\sigma_{\mathrm{ph}}\left(V_{\mathrm{GS}}\right)^{30}$, the optimization of the mixing efficiency $\left(\Delta_{R_{\text {ext }}}\right)$ requires $\frac{d \sigma_{\mathrm{oh}}}{d V_{\mathrm{GS}}}$ to be maximized. $\sigma_{\mathrm{ph}}\left(V_{\mathrm{GS}}\right)$ depends on several factors, such as the residual charge carrier density, $n_{0}{ }^{63}$, and the dominating scattering mechanisms ${ }^{63,67}$. So, it depends on SLG quality and its
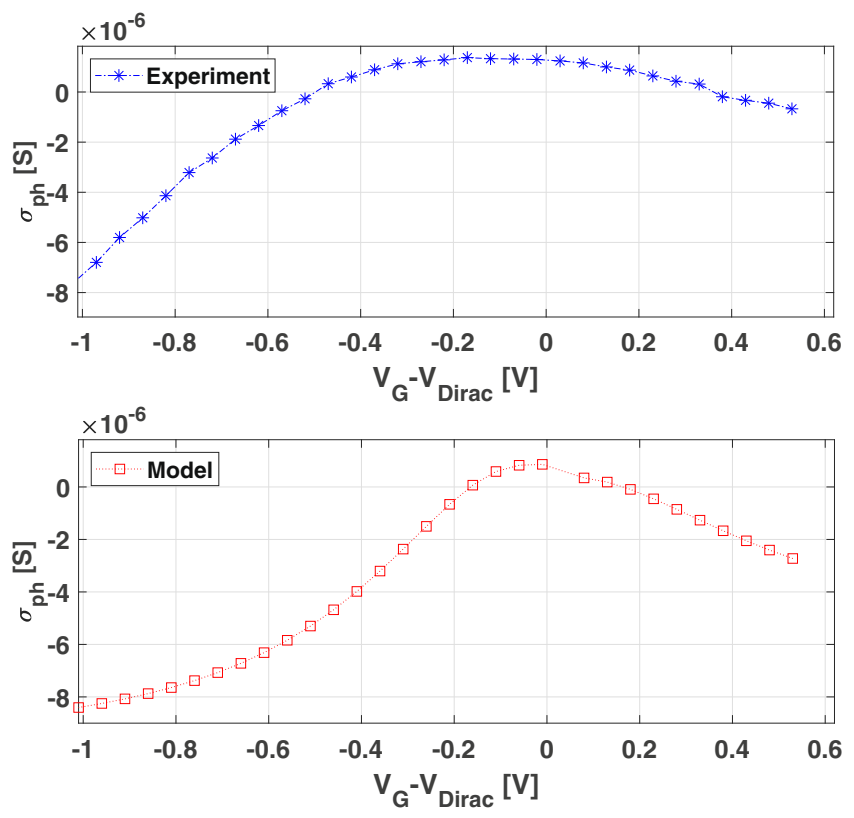

Fig. 7 Comparison between measured and calculated photoconductivity. a Measured and $\mathbf{b}$ calculated photoconductivity of illuminated SLG channel.

dielectric environment. We compute $\sigma_{\mathrm{ph}}\left(V_{\mathrm{GS}}\right)$ from the Drude model for free carrier conductivity in $\mathrm{SLG}^{63}$ :

$$
\sigma\left(T_{e}, \mu_{c}\right)=\frac{D\left(T_{e}, \mu_{c}\right)}{\pi \Gamma\left(T_{e}, \mu_{c}\right)}
$$

where $T_{e}$ is the electron temperature, $\Gamma$ is the scattering rate, $D$ is the Drude weight ${ }^{63,67}$, and $\mu_{c}$ is the chemical potential. We calculate $\mu_{c}$ (see Methods for details), and then the effect of $e-h$ puddles by replacing $\mu_{c}$ with $\mu_{c} \rightarrow \sqrt[4]{\mu_{c}^{4}+\Delta \mu_{\text {puddles }}} 63$, where $\Delta_{\mu_{\text {puddles }}}$ is the energy width of the puddles region ${ }^{63}$. The photoconductivity due to illumination of the SLG channel at a given $\mu_{c}$ is then given by ${ }^{63}$ :

$$
\sigma_{\mathrm{ph}}=\sigma\left(T_{\text {light }}\right)-\sigma\left(T_{\text {dark }}\right)=\frac{D\left(T_{\text {light }}\right)}{\pi \Gamma\left(T_{\text {light }}\right)}-\frac{D\left(T_{\text {dark }}\right)}{\pi \Gamma\left(T_{\text {dark }}\right)}
$$

where $T_{\text {light }}$ is the hot-electron temperature of the illuminated SLG and $T_{\text {dark }}$ is the electron temperature in dark, see Methods for details. Figure 7 plots the measured photoconductivity (blue) and the computed one (red) using Eq. (7). We calculate $\Delta \mu_{\text {puddles }}$ from $n_{0}$, extracted from the experimental DC measurement of the conductivity (see Methods). We calculate $\Gamma$ from the measured $\mu$ (see Methods). We get $\Gamma \sim 50 \mathrm{meV}$, which corresponds to a scattering 
time $\tau \sim 80$ fs at $\mathrm{E}_{F} \sim 200 \mathrm{meV}$, at the maximum $V_{\mathrm{GS}}$, in agreement with experiments.

We then calculate $\frac{d \sigma_{\mathrm{ph}}}{d V_{\mathrm{GS}}}$ to find the maximum downconversion efficiency. This can be increased by $\sim 27 \mathrm{~dB}$ (power unit) for SLG with $\mu_{\mathrm{fe}} \sim 30,000 \mathrm{~cm}^{2} \mathrm{~V}^{-1} \mathrm{~s}^{-1}(\tau=1 / \Gamma \sim 0.6 \mathrm{ps})$ and $n_{0} \sim 10^{11} \mathrm{~cm}^{-2}$, see Methods. Thus, the control of the transition between the two different photocurrent mechanisms could lead to a maximization of $\Delta_{R}$ and, in turn, a maximization of downconversion efficiency.

The $3 \mathrm{~dB}$ BW is $\sim 19.7 \mathrm{GHz}$ when operated as OEM, Fig. 4 . This behavior is modeled in Eq. (3) by including the factor $\beta\left(f_{\mathrm{RF}}\right)$. To understand the optoelectronic-mixing dependence on $f_{\mathrm{RF}}$ and, thus, on $f_{\mathrm{IF}}$ ( $f_{\text {opt }}$ being fixed), we consider a typical figure of merit of high-frequency transistors: the transducer power gain ${ }^{5}$, defined as: $\mathrm{G}_{T}=\frac{P_{\text {load }}}{P_{\text {avs }}}$, where $P_{\text {load }}$ is the power delivered to the load and $P_{\text {avs }}$ is the source power. $G_{T}$ coincides with the modulus of the $S_{21}$ parameter when source and load are matched ${ }^{5}$. This is the case in our measurements, where the power is delivered from the VNA
$50 \Omega$-source and measured on a $50 \Omega$ receiver. $G_{T}$ is close to the $\mathrm{S}_{21}$ parameters. An external impedance matching could increase the downconversion efficiency by maximizing the power delivered by the GFET, other than increasing BW. We do not observe saturation in the photodetected signal at the highest optical power available in our setup $(60 \mathrm{~mW})$. Thus, illuminating a wider channel surface, while maintaining the same optical power density, should increase the downconversion efficiency. An enhancement of SLG-light interaction can also improve the downconversion efficiency. In all, $\sim 70 \%$ absorption could be achieved by integrating SLG on a waveguide ${ }^{68}$. This could enhance the downconversion efficiency by $\sim 30 \mathrm{~dB}$, compared with the normal incidence case $\left(\sim 2.3 \%\right.$ absorption $\left.{ }^{23}\right)$.

In summary, we reported high-frequency graphene transistors operating as OEMs for frequencies up to at least $67 \mathrm{GHz}$. The photodetection BW exceeds $67 \mathrm{GHz}$. The BW of the devices operated as OEMs is $19.7 \mathrm{GHz}$. The conversion efficiency is at
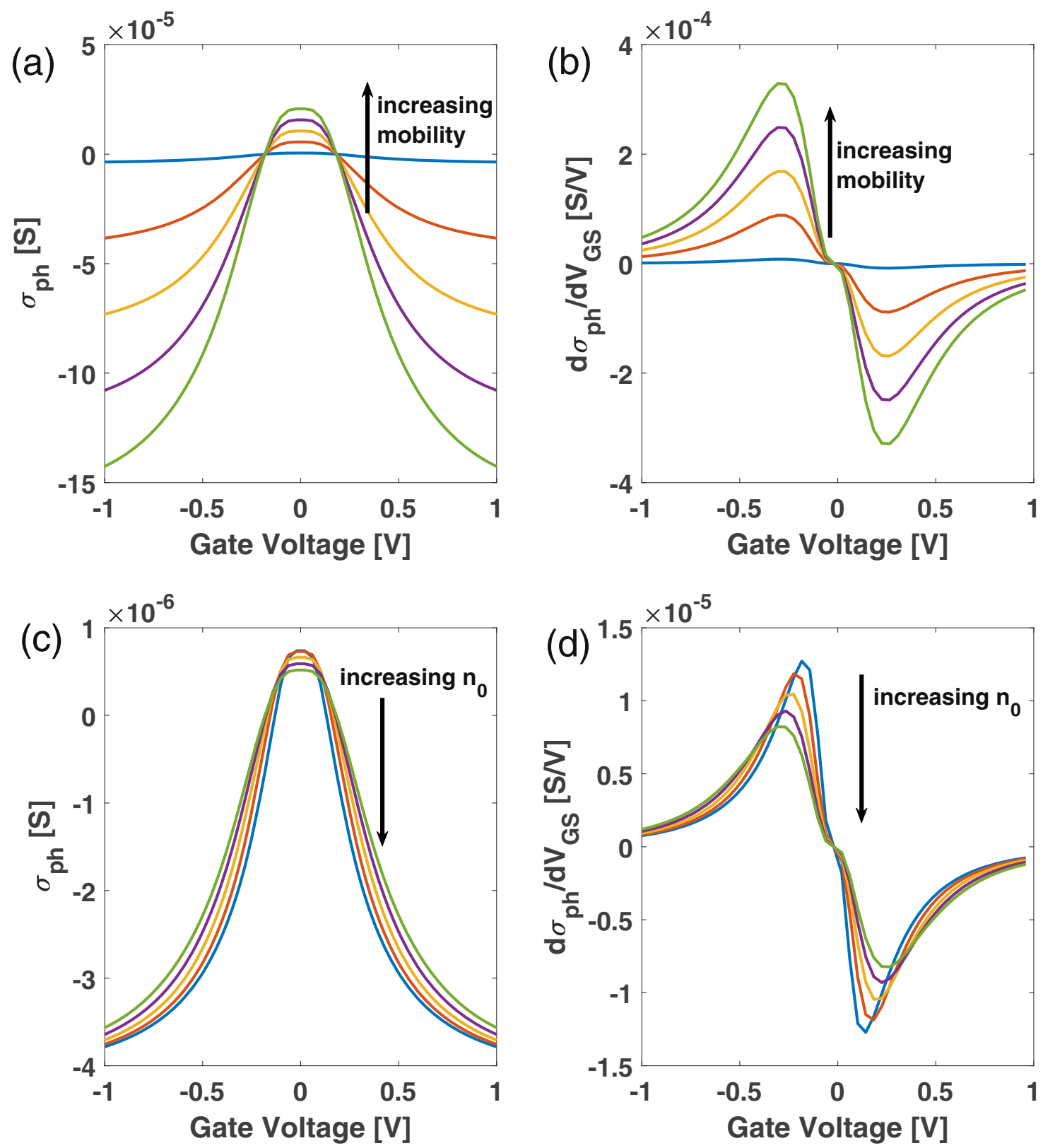

Fig. 8 Optoelectronic-mixing performance. a Calculated photoconductivity and b photoconductivity derivative for $\mu_{\mathrm{fe}}$ varying from 3800 to $100,000 \mathrm{~cm} 2$ $\mathrm{V}^{-1} \mathrm{~s}^{-1}$. c Calculated photoconductivity and $\mathbf{d}$ photoconductivity derivative for $n_{0}$ varying from $10^{11}$ to $4.5 \cdot 10^{11} \mathrm{~cm}^{-2}$. 
least two orders of magnitude higher than previous graphene $\mathrm{OEMs}^{29}$. It can be further increased using high-quality samples with $\mu \sim 10,000-100,000 \mathrm{~cm}^{2} \mathrm{~V}^{-1} \mathrm{~s}^{-1}$ and increasing light-matter interaction. This can increase the downconversion efficiency $>50$ $\mathrm{dB}$, overcoming state-of the-art performances of OEMs based on any other technology ${ }^{31}$. Our frequency operation is already comparable with state-of-the-art performance of OEMs based on any other technology ${ }^{31}$. Thus, our work paves the way for the use of graphene-based transistors as OEMs in applications exploiting mm-waves, such as telecommunications and RADAR/LIDAR.

\section{Methods}

Photocurrent modeling. Equations 1-5 show that the optoelectronic-mixing efficiency is proportional to $\Delta_{R_{\text {ext }}}$, which can be expressed as:

$$
\Delta_{R_{\text {ext }}}=\left.\beta\left(f_{\mathrm{RF}}\right) \frac{d R_{\mathrm{ext}}\left(V_{\mathrm{GS}}\right)}{d V_{\mathrm{GS}}}\right|_{V_{\mathrm{GS}}=\bar{V}_{\mathrm{GS}}}
$$

Since $\mathrm{R}_{\text {ext }}$ is proportional to $\sigma_{\mathrm{ph}}\left(V_{\mathrm{GS}}\right)^{30}$, the optimization of the mixing efficiency requires $\frac{d \sigma_{\mathrm{ph}}}{d V_{\mathrm{GS}}}$ to be maximized. The mixing efficiency can be related via $\Delta_{R_{\text {ext }}}$ to SLG's $\mu$ and $n^{63,67}$. The Drude model for free carrier conductivity in SLG gives ${ }^{63}$ :

$$
\sigma\left(\omega, T_{e}\right)=\frac{D\left(T_{e}\right)}{\pi\left[\Gamma\left(T_{e}\right)-i \omega\right]}
$$

In the case of Dirac Fermions the Drude weight is ${ }^{63}$ :

$$
D\left(T_{e}\right)=\frac{2 e^{2}}{\hbar^{2}} k_{B} T_{e} \ln \left[2 \cosh \left(\frac{\mu_{c}\left(T_{e}\right)}{2 k_{B} T_{e}}\right)\right]
$$

with $k_{B}$ the Boltzmann constant. In the $\mathrm{GHz}$ range, $\omega \sim 10^{9}-10^{10} \mathrm{rad} / \mathrm{s}$. This value is negligible compared to our $\Gamma$, which lies in the range $10^{12}-10^{13} \mathrm{rad} / \mathrm{s}^{32}$. The photoconductivity owing to the illumination of the SLG channel is then ${ }^{63}$ :

$$
\sigma_{\text {ph }}=\sigma\left(T_{\text {light }}\right)-\sigma\left(T_{\text {dark }}\right)=\frac{D\left(T_{\text {light }}\right)}{\pi \Gamma\left(T_{\text {light }}\right)}-\frac{D\left(T_{\text {dark }}\right)}{\pi \Gamma\left(T_{\text {dark }}\right)}
$$

This depends on $T$, as experimentally shown in ref. ${ }^{34}$. Increasing $T$ decreases the photodetection efficiency ${ }^{34}$. This is consistent with the screening reduction of the scattering mechanism while increasing $\mathrm{T}_{e}{ }^{32}$, since a smaller $T$ change between dark and illumination conditions takes place.

$\mu_{c}$ is $T$-dependent. It decreases while increasing $T$ to keep the number of conduction band carriers constant ${ }^{69}$. So, it is lower in illumination conditions with respect to dark. To account for this, we compute $\mu_{c}$ by numerical inversion of the following $T$-dependent formula ${ }^{60,63}$ :

$$
\frac{2}{\pi} \frac{\left(k_{B} T\right)^{2}}{\left(\hbar v_{0}\right)^{2}}\left[L i_{2}\left(-e^{\frac{-\mu_{c}}{k_{B} T}}\right)-L i_{2}\left(-e^{\frac{\mu_{c}}{k_{B} T}}\right)\right]=\frac{C_{\mathrm{ox}} V_{\mathrm{GS}}}{e}-\frac{\mu_{c} C_{\mathrm{ox}}}{e^{2}}
$$

In Eq. (12), $C_{\mathrm{ox}}$ is the geometrical gate capacitance per unit area, $v_{0}$ is the Fermi velocity and $L i_{2}$ is the dilogarithm function. This is valid in low and high doping and also includes the effects of quantum capacitance. $\Gamma$ is calculated from $\mu_{f e} \sim$ $2500 \mathrm{~cm}^{2} /(\mathrm{Vs})$ using ${ }^{16}$ :

$$
\Gamma=\frac{1}{\tau}=\frac{e v_{F}^{2}}{\mu_{c}\left(T_{e}\right) \mu_{\mathrm{fe}}}
$$

We get $\Gamma \sim 80 \mathrm{meV}$, i.e. $\tau \sim 50$ fs for $\mu_{c} \sim 0.2 \mathrm{eV}$. Charge puddles are taken into account by replacing $\mu_{c}$ with ${ }^{63}$ :

$$
\mu_{c} \rightarrow \sqrt[4]{\mu_{c}^{4}+\Delta \mu_{\text {puddles }}^{4}}
$$

$\Delta \mu_{\text {puddles }}$ is calculated as ${ }^{60}$ :

$$
\Delta \mu_{\text {puddles }}=\sqrt{n_{0} \pi} \hbar v_{F} \sim 120 \mathrm{meV}
$$

Here, $n_{0} \sim 5 \cdot 10^{11} \mathrm{~cm}^{-2}$ extracted from the measured DC minimum of conductivity $\sigma_{\min }{ }^{60}$ :

$$
n_{0}=\frac{\sigma_{\min }}{e \mu_{\mathrm{fe}}}
$$

From the DC measurements, we get $C_{o x} \sim 4 f F / \mu \mathrm{m}^{2}$. The experimental curve in Fig. 7 uses $\mathrm{T}_{e}$ as a fitting parameter, getting $T_{e} \sim 320 \mathrm{~K}$ in the laser spot region.

Optoelectronic mixing efficiency. We now evaluate the effects of $\mu$ and $n_{0}$ on mixing efficiency. Since our SLG is substrate-supported and not encapsulated in hBN, long-range scattering limits conductivity ${ }^{32}$, more specifically Coulomb scattering $32,70,71$. In this regime, the typical $\tau$ is in the range of hundreds of $\mathrm{fs}^{32}$. Highquality SLG can have low $n_{0}\left(<10^{12} \mathrm{~cm}^{-2}\right)$ and high $\left.\mu>100,000 \mathrm{~cm}^{2} \mathrm{~V}^{-1} \mathrm{~s}^{-1}\right)^{50}$. In high-quality SLG encapsulated with $\mathrm{hBN}$, as in ref. ${ }^{50}$, transport is dominated by random-strain fluctuations ${ }^{33,72}$. For such SLG, a change in $\mu<20 \%$ was measured as a function of $n^{50}$. In order to estimate the performance in such SLG, we assume no dependence of $\mu$ o $n$. Within this assumption, $\tau \propto \mu$ for $\mu \gg K_{B} T^{60}$, so Eq. (13) is still valid. For $\mu \sim 100,000 \mathrm{~cm}^{2} \mathrm{~V}^{-1} \mathrm{~s}^{-1}, \tau$ can be up to $2 \mathrm{ps}$ for $\mathrm{E}_{F} \sim 0.2 \mathrm{eV}^{16}$. We thus calculate $\sigma_{\mathrm{ph}}$ for $\mu$ up to $100,000 \mathrm{~cm}^{2} \mathrm{~V}^{-1} \mathrm{~s}^{-1}$ and $n_{0} \sim 5 \cdot 10^{11} \mathrm{~cm}^{-2}$, Fig. 8a. By comparing the green and the blue curves, which show the photoconductivity for $\mu \sim 100,000$ and $2.500 \mathrm{~cm}^{2} \mathrm{~V}^{-1} \mathrm{~s}^{-1}$, we predict an increase $\sim 40$ times at both low (i.e. $V_{\mathrm{GS}}=0$ ) and high electrostatic doping (i.e., for $V_{G S}=1 \mathrm{~V}$ ). We then differentiate the curves in Fig. 8a. Figure $8 \mathrm{~b}$ shows an increase in $d \sigma_{\mathrm{ph}} / d V_{\mathrm{GS}}$, (i.e. an increase of $\Delta_{R_{\text {ext }}}$ ) of a factor $\sim 40$.

Another parameter that can be improved by employing high-quality SLG is $n_{0}$. Fig. $8 \mathrm{c}$ plots the photoconductivity for $n_{0}$ between $10^{11} \mathrm{~cm}^{-2}$ (corresponding to highquality $\mathrm{SLG}^{50}$ ) and $5 \times 10^{11} \mathrm{~cm}^{-2}$ (our case), while keeping $\mu_{\mathrm{fe}}=2500 \mathrm{~cm}^{2} \mathrm{~V}^{-1} \mathrm{~s}^{-1}$ In this case, the photoconductivity increases by a factor $\sim 1.4$ near the CNP, as well as at high doping $\left(V_{G S}=1 \mathrm{~V}\right)$, with $d \sigma_{\mathrm{ph}} / d V_{\mathrm{GS}}$ almost doubled, Fig. 8d. This means that the voltage operating point of the device can be decreased.

Our prediction is based on the model presented in ref. ${ }^{32}$, which indicates the screening reduction of the Coulomb impurity scattering mechanism while increasing $\mathrm{T}^{32}$. For Coulomb scattering in samples with $\mu_{f e}$ up to $1000 \mathrm{~cm}^{2} \mathrm{~V}^{-1} \mathrm{~s}^{-171}$, this is supported by several experimental and theoretical works ${ }^{32,63,67}$. For samples with ultrahigh $\mu_{\mathrm{fe}}$ up to $100,000 \mathrm{~cm}^{2} \mathrm{~V}^{-1} \mathrm{~s}^{-150}$ ), the dominant mechanism limiting $\mu_{\mathrm{fe}}$ is strain disorder ${ }^{33}$, with two contributions: (i) random scalar potential and (ii) random gauge potential $^{33}$. The first is sensitive to $\mathrm{T}$ increase owing to screening reduction ${ }^{33}$, similar to what happens for Coulomb scattering ${ }^{32}$, while the second is not ${ }^{33}$. Thus, for ultra-highquality $\mu_{\mathrm{fe}}$ samples, the "screening reduction" picture may not be accurate. Therefore, we use an intermediate value $\mu_{\mathrm{fe}}=30,000 \mathrm{~cm}^{2} \mathrm{~V}^{-1} \mathrm{~s}^{-1}$, between $10,000 \mathrm{~cm}^{2} \mathrm{~V}^{-1} \mathrm{~s}^{-1}$ (i.e., Coulomb scattering regime ${ }^{71}$ ) and $100,000 \mathrm{~cm}^{2} \mathrm{~V}^{-1} \mathrm{~s}^{-1}$ (i.e., random-strain fluctuations-induced scattering regime ${ }^{33}$ ) to infer the performance boost of OEMs based on SLG FET owing to $\mu_{\mathrm{fe}}$ increase. In this case, for $n_{0}=10^{11} \mathrm{~cm}^{-2}$, we predict an increase of $d \sigma_{\mathrm{ph}} / d V_{\mathrm{GS}}$ by a factor $\sim 23 \mathrm{~dB}$. Thus, the downcoversion efficiency in power units can increase $\sim 27 \mathrm{~dB}$, overcoming the OEMs state-of-the-art performance ${ }^{12,31}$.

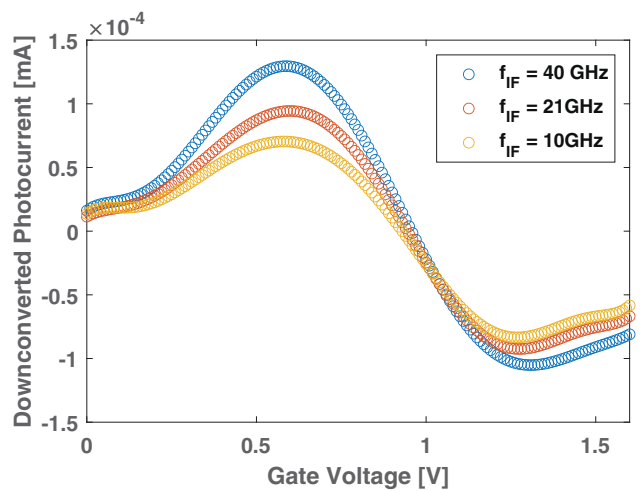

Fig. 9 Downconverted photocurrent versus gate voltage at different frequencies. The three curves show the downconverted photocurrent at 10, $21,40 \mathrm{GHz}$. The same behavior shown in Fig. 6 is observed, regardless of the operating frequency.

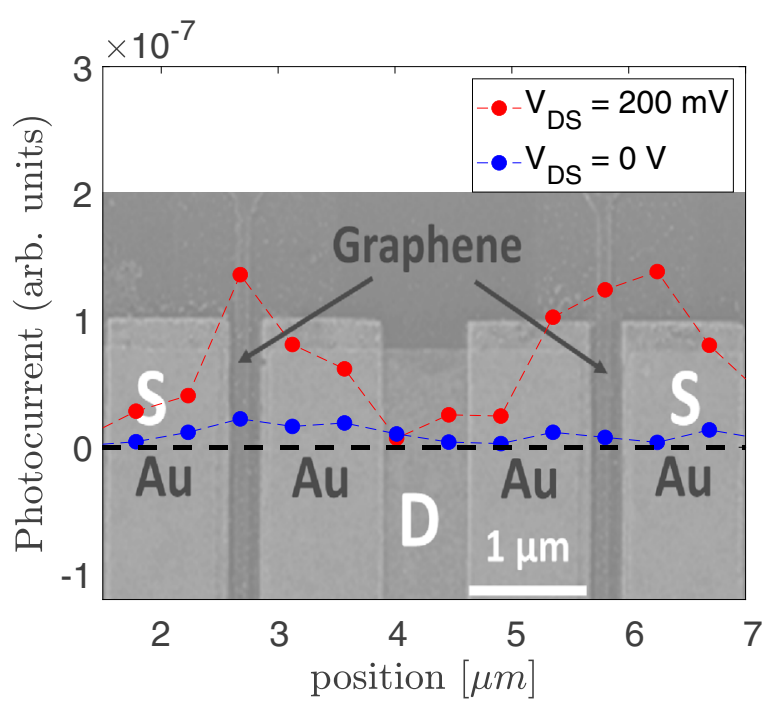

Fig. 10 Photoresponse as a function of laser spot position. The red and blue curves show the measured photoresponse as a function of laser spot position along the black dashed cut line, at $200 \mathrm{mV}$ and $\mathrm{OV}$, respectively. 
Fig. 9 plots the downconverted photocurrent at three frequencies as a function of $\mathrm{V}_{\mathrm{GS}}$. This shows Fig. 6 is valid regardless of operating frequency.

Dependence of the laser spot position on photoresponse. Figure 10 shows the photoresponse as a function of laser spot position over the device, along the dashed black line. The photocurrent is measured with the VNA used for the RF measurements in Fig. 2a. The optical beam intensity is modulated at $10 \mathrm{GHz}$ and the optical power impinging the sample is $\sim 10 \mathrm{~mW}$. The beam scan step is $\sim 300 \mathrm{~nm}$, smaller than the spot size of the laser $\sim 2 \mu \mathrm{m}$, as defined by the FWHM of the intensity of the Gaussian laser profile. The red dots represent the measured photocurrent at $V_{D S}=0.2 \mathrm{~V}$, whereas the blue ones are for $V_{\mathrm{DS}}=0 \mathrm{~V}$. The maximum photocurrent is registered when $V_{\mathrm{DS}}=0.2 \mathrm{~V}$ and the laser spot is over the GFET channel. We observe very low photocurrent, comparable with the instrument noise floor ( - $90 \mathrm{dBm}$ electrical power detected over the internal $50 \Omega$ impedance of the instrument) at $V_{\mathrm{DS}}=0$. At $V_{\mathrm{DS}}=0.2 \mathrm{~V}$, we do not observe a change of the photocurrent sign (due to, e.g., asymmetrical heating effect $^{73}$ ) when the laser spot is scanned from the source to the drain contact. Thus, the role of contacts in the photocurrent generation can be neglected.

\section{Data availability}

The data that support the findings of this study are available from the corresponding author upon reasonable request.

Received: 13 June 2019; Accepted: 29 March 2021;

Published online: 12 May 2021

\section{References}

1. Maas, S. A. Microwave Mixers (Artech House Inc., 1986).

2. Gagliardi, R. M. \& Karp, S. Optical communications (Wiley-Interscience, 1976).

3. $\mathrm{Gu}, \mathrm{Q}$. RF system design of transceivers for wireless communications (Springer, 2006).

4. Skolnik, M. I. Introduction to radar systems (McGraw-Hill, 2001).

5. Pozar, D. M. Microwave engineering (John Wiley \& Sons, 2009).

6. Chizh, A. \& Malyshev, S. Fiber-optic system for local-oscillator signal distribution in active phased arrays. 11th European Radar Conference (2014).

7. Ruff, W. C. et al. Self-mixing detector candidates for an $\mathrm{FM} / \mathrm{cw}$ ladar architecture. SPIE Proceedings, 4035 (2000.

8. Pillet, G., Morvan, L., Dolfi, D. \& Huignard, J.-P. Wideband dual-frequency lidar-radar for high-resolution ranging, profilometry, and Doppler measurement. Proceedings Volume 7114, Electro-Optical Remote Sensing, Photonic Technologies, and Applications II; 71140E (2008).

9. Ghelfi, P. et al. A fully photonics-based coherent radar system. Nature 507, 341 (2014).

10. Vercesi, V. et al. Frequency-agile dual-frequency lidar for integrated coherent radar-lidar architectures. Opt. Lett. 40, 1358 (2015).

11. Choi, C.-S. et al. 60-GHz bidirectional radio-on-fiber links based on InPInGaAs HPT optoelectronic mixers. IEEE Photonics Technol. Lett. 17, 2721 (2005).

12. Rouvalis, E., Fice, M. J., Renaud, C. C. \& Seeds, A. J. Optoelectronic detection of millimetre-wave signals with travelling-wave uni-travelling carrier photodiodes. Opt. Express 19, 2079 (2011).

13. Sze, S. M. \& Ng, K. K. Physics of semiconductor devices (John Wiley \& Sons, 2006).

14. Bonaccorso, F., Sun, Z., Hasan, T. \& Ferrari, A. C. Graphene photonics and optoelectronics. Nat. Photonics 4, 611 (2010)

15. Koppens, F. H. L. et al. Photodetectors based on graphene, other twodimensional materials and hybrid systems. Nat. Nanotechnol. 9, 780 (2014).

16. Romagnoli, M. et al. Graphene-based integrated photonics for next-generation datacom and telecom. Nat. Rev. Mater. 3, 392 (2018).

17. Sun, Z. P. et al. Graphene mode-locked ultrafast laser. ACS Nano 4, 803 (2010).

18. Tang, Z., Li, Y., Yao, J. \& Pan, S. Photonics based microwave frequency mixing: methodology and applications. Laser Photonics Rev. 14, 1 (2019).

19. Purdie, D. G. et al. Cleaning interfaces in layered materials heterostructures. Nat. Commun. 9, 5387 (2018).

20. Breusing, $M$. et al. Ultrafast nonequilibrium carrier dynamics in a single graphene layer. Phys. Rev. B 83, 153410 (2011).

21. Brida, D. et al. Ultrafast collinear scattering and carrier multiplication in graphene. Nat. Commun. 4, 1987 (2013)

22. Tomadin, A., Brida, D., Cerullo, G., Ferrari, A. C. \& Polini, M. Nonequilibrium dynamics of photoexcited electrons in graphene: collinear scattering, Auger processes, and the impact of screening. Phys. Rev. B 88, 035430 (2013)

23. Nair, R. R. et al. Fine structure constant defines visual transparency of graphene. Science 320, 1308 (2008)
24. Pospischil, A. et al. CMOS-compatible graphene photodetector covering all optical communication bands. Nat. Photonics 7, 892 (2013).

25. Thomas, S. CMOS-compatible graphene. Nat. Electron. 1, 612 (2018).

26. Giambra, M. A. et al. Wafer-scale integration of graphene-based photonic devices. ACS Nano 15, 3171 (2021).

27. Mzali, S. et al. Stabilizing a graphene platform toward discrete components. Appl. Phys. Lett. 109, 253110 (2016).

28. Mao, X. et al. Optoelectronic mixer based on graphene FET. IEEE Elec. Dev. Lett. 36, 253-255 (2015).

29. Montanaro, A. et al. Thirty gigahertz optoelectronic mixing in chemical vapor deposited graphene. Nano Lett. 16, 2988 (2016).

30. Saleh, B. E. A. \& Teich, M. C. Fundamentals of photonics, 3rd edn. (Wiley Series in Pure and Applied Optics, 2015).

31. Mohammad, A. W. et al. 5 Gbps wireless transmission link with an optically pumped uni-traveling carrier photodiode mixer at the receiver. Opt. Express 26, 2884-2890 (2018)

32. Tomadin, A. et al. The ultrafast dynamics and conductivity of photoexcited graphene at different Fermi energies. Sci. Adv. 4, 5313 (2018).

33. Couto, N. J. G. et al. Random strain fluctuations as dominant disorder source for high-quality on-substrate graphene devices. Phys. Rev. X 4, 041019 (2014).

34. Freitag, M., Low, T., Xia, F. N. \& Avouris, P. Photoconductivity of biased graphene. Nat. Photonics 7, 53 (2013).

35. Ferrari, A. C. et al. Science and technology roadmap for graphene, related twodimensional crystals, and hybrid systems. Nanoscale 7, 4598 (2015).

36. Sassi, U. et al. Graphene-based mid-infrared room-temperature pyroelectric bolometers with ultrahigh temperature coefficient of resistance. Nat. Commun. 8, 14311 (2017)

37. Li, X. S. et al. Large-area synthesis of high-quality and uniform graphene films on copper foils. Science 324, 1312 (2009).

38. Lagatsky, A. A. et al. $2 \mu \mathrm{m}$ solid-state laser mode-locked by single-layer graphene. Appl. Phys. Lett. 102, 013113 (2013).

39. Ferrari, A. C. \& Basko, D. M. Raman spectroscopy as a versatile tool for studying the properties of graphene. Nat. Nanotechnol. 8, 235 (2013).

40. Cancado, L. G. et al. Quantifying defects in graphene via raman spectroscopy at different excitation energies. Nano Lett. 11, 3190 (2011).

41. Ferrari, A. C. et al. Raman spectrum of graphene and graphene layers. Phys. Rev. Lett. 97, 187401 (2006).

42. Bae, S. et al. Roll-to-roll production of 30-inch graphene films for transparent electrodes. Nat. Nanotechnol. 5, 574 (2010).

43. Bonaccorso, F. et al. Production and processing of graphene and $2 \mathrm{~d}$ crystals. Mater. Today 15, 564 (2012).

44. Casiraghi, C. et al. Rayleigh imaging of graphene and graphene layers. Nano Lett. 7, 2711 (2007).

45. Klar, P. et al. Raman scattering efficiency of graphene. Phys. Rev. B 87, 205435 (2013).

46. Das, A. et al. Monitoring dopants by Raman scattering in an electrochemically top-gated graphene transistor. Nat. Nanotechnol. 3, 210 (2008).

47. Basko, D. M., Piscanec, S. \& Ferrari, A. C. Electron-electron interactions and doping dependence of the two-phonon Raman intensity in graphene. Phys. Rev. B 80, 165413 (2009).

48. Bruna, M. et al. Doping dependence of the raman spectrum of defected graphene. ACS Nano 8, 7432 (2014).

49. $\mathrm{Li}, \mathrm{X}$. et al. Large-area graphene single crystals grown by low-pressure chemica vapor deposition of methane on copper. J. Am. Chem. Soc. 133, 2816 (2011)

50. De Fazio, D. et al. High-mobility, wet-transferred graphene grown by chemical vapor deposition. ACS Nano 13, 8926 (2019).

51. $\mathrm{Wu}, \mathrm{Y}$. et al. State-of-the-art graphene high-frequency electronics. Nano Lett. 6, 3062 (2012).

52. Petrone, N., Meric, I., Hone, J. \& Shepard, K. L. Graphene field-effect transistors with gigahertz-frequency power gain on flexible substrates. Nano Lett. 13, 121 (2012).

53. Rzin, M. et al. Impact of gate-drain spacing on low-frequency noise performance of in situ sin passivated InAlGaN/GaN MIS-HEMTs. IEEE Trans. Electron. Devices 64, 2820 (2017).

54. Schwierz, F. Graphene transistors: status, prospects, and problems. Proc. IEEE 101, 1567 (2013)

55. Wei, W. et al. Graphene FETs with aluminum bottom-gate electrodes and its natural oxide as dielectrics. IEEE Trans. Elec. Dev. 62, 2769 (2015).

56. Schwierz, F., Pezoldt, J. \& Granzner, R. Two-dimensional materials and their prospects in transistor electronics. Nanoscale 7, 8261 (2015).

57. Guo, Z. et al. Ultrafast photoconductivity of graphene nanoribbons and carbon nanotubes. Nano Lett. 13, 942 (2013).

58. Davidson, A., Jones, K. \& Strid, E. LRM and LRRM calibrations with automatic determination of load inductance, 36th ARFTG conference digest. IEEE 18, 57-63 (1990).

59. Inagaki, K., Kawanishi, T. \& Izutsu, M. Optoelectronic frequency response measurement of photodiodes by using high-extinction ratio optical modulator. IEICE Elec. Exp. 9, 220 (2012). 
60. Zebrev, G. I. Graphene field effect transistors: diffusion-drift theory. IntechOpen $\mathbf{2 3}$ https://www.fzu.cz/ knizek/literatura/Ashcroft_Mermin.pdf (2011)

61. Lemme, M. C., Echtermeyer, T. J., Baus, M. \& Kurz, H. A graphene field-effect device. IEEE Electron Device Lett. 28, 282 (2007).

62. Ashcroft, N. W. \& Mermin, N. D. Holt. Solid State Physics. (Rinehart and Winston, US, 1976).

63. Frenzel, A. J., Lui, C. H., Shin, Y. C., Kong, J. \& Gedik, N. Semiconducting-tometallic photoconductivity crossover and temperature-dependent drude weight in graphene. Phys. Rev. Lett. 113, 056602 (2014).

64. De Fazio, D. et al. High responsivity, large-area graphene/MoS2 flexible photodetectors. ACS Nano 10, 8252 (2016).

65. Mueller, T., Xia, F. \& Avouris, P. Graphene photodetectors for high-speed optical communications. Nat. Photonics 4, 297-301 (2010).

66. Sedra, A. S. \& Smith, K. C. Microelectronic Circuits, 5th edn. (Oxford University Press, 2019)

67. Shi, S.-F. et al. Controlling graphene ultrafast hot carrier response from metallike to semiconductor-like by electrostatic gating. Nano Lett. 14, 1578 (2014).

68. Schuler, S. et al. Controlled generation of a p-n junction in a waveguide integrated graphene photodetector. Nano Lett. 16, 7107 (2016).

69. Jensen, S. A. et al. Competing ultrafast energy relaxation pathways in photoexcited graphene. Nano Lett. 14, 5839-5845 (2014).

70. Tan, Y.-W. et al. Measurement of scattering rate and minimum conductivity in graphene. Phys. Rev. B 99, 246803 (2007).

71. Das Sarma, S., Adam, S., Hwang, E. H. \& Rossi, E. Electronic transport in twodimensional graphene. Rev. Mod. Phys. 83, 407 (2011).

72. Katsnelson, M. I. \& Geim, A. K. Electron scattering on microscopic corrugations in graphene. Phil. Trans. R. Soc. A. 366, 195-204 (2007).

73. Tielrooij, K. J. et al. Hot-carrier photocurrent effects at graphene-metal interfaces. Phys. Condens. Matter 27, 164207 (2015).

\section{Acknowledgements}

We acknowledge funding from EU Graphene Flagship (Graphene Core2 and Core3 under grant agreement No 785219 and 881603), the French RENATECH network, the University Lille CMNF platform, ERC Grants Hetero2D and MINERGRACE, EPSRC Grants EP/K01711X/1, EP/K017144/1, EP/ N010345/1, EP/L016087/1.

\section{Author contributions}

A.M. and W.W. performed the optoelectronic, DC, and RF characterization. A.M. and P.A. carried out the device simulations, W.W. fabricated the device, D.D.F., U.S., G.S., A.C.F. grew, transferred, and characterized the graphene samples. A.C.F., P.L., H.H. supervised the work. A.M., P.L., E.P. conceived the idea. A.M. wrote the paper, with contributions from all authors.

\section{Competing interests}

The authors declare no competing interests.

\section{Additional information}

Correspondence and requests for materials should be addressed to E.P.

Peer review information Nature Communications thanks Ryan Suess and the other, anonymous reviewer(s) for their contribution to the peer re-view of this work.

Reprints and permission information is available at http://www.nature.com/reprints

Publisher's note Springer Nature remains neutral with regard to jurisdictional claims in published maps and institutional affiliations.

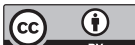

Open Access This article is licensed under a Creative Commons Attribution 4.0 International License, which permits use, sharing, adaptation, distribution and reproduction in any medium or format, as long as you give appropriate credit to the original author(s) and the source, provide a link to the Creative Commons license, and indicate if changes were made. The images or other third party material in this article are included in the article's Creative Commons license, unless indicated otherwise in a credit line to the material. If material is not included in the article's Creative Commons license and your intended use is not permitted by statutory regulation or exceeds the permitted use, you will need to obtain permission directly from the copyright holder. To view a copy of this license, visit http://creativecommons.org/ licenses/by/4.0/.

(c) The Author(s) 2021, corrected publication 2021 